\title{
Efforts sur une conduite sous-marine en zone côtière
}

\author{
Christophe Chevalier
}

Docteur, M2C - Groupe Mécanique des Fluides, Université de Caen

Eloi Lambert

Maître de Conférences, IUFM, Université de Rouen

Michel Bélorgey

Professeur, M2C - Groupe Mécanique des Fluides, Université de Caen

\section{Résumé}

Nous avons mesuré les efforts exercés sur un cylindre horizontal près du fond par l'action de la houle se propageant vers la côte. Celle-ci est modélisée par une plage de pente constante qui va induire une déformation progressive de la houle. Les efforts mesurés sont influencés par la hauteur et la dissymétrie de la houle ainsi que par l'écartement du cylindre par rapport au fond. Une modélisation de l'effort en ligne par la formulation de Morison est également menée et les coefficients hydrodynamiques correspondants sont présentés. Nous montrons ainsi que l'emploi d'un modèle simplifié de la vitesse horizontale, prenant en compte la dissymétrie de l'écoulement, permet d'étendre la validité du modèle de Morison aux houles pré-déferlantes.

\begin{abstract}
We have measured wave forces on a horizontal cylinder near the seabed in the coastal zone. A beach of constant slope induces the progressive wave deformation. The measured forces are influenced by the wave height, the wave asymmetry and the distance between the cylinder and the bottom. Modelling of the in-line force using the Morison formulation is also performed and the resulting hydrodynamic coefficients are presented. We show that the use of a horizontal velocity model taking into account the flow asymmetry enables to extend the validity of the Morison model to shoaling waves.
\end{abstract}

\section{Introduction}

La stabilisation d'une conduite sous-marine à proximité ou sur le fond est un problème très complexe du fait de la multitude d'éléments à considérer. Il est impératif de bien connaître la nature exacte des différentes forces s'exerçant sur uine telle structure afin d'en assurer le dimensionnement.
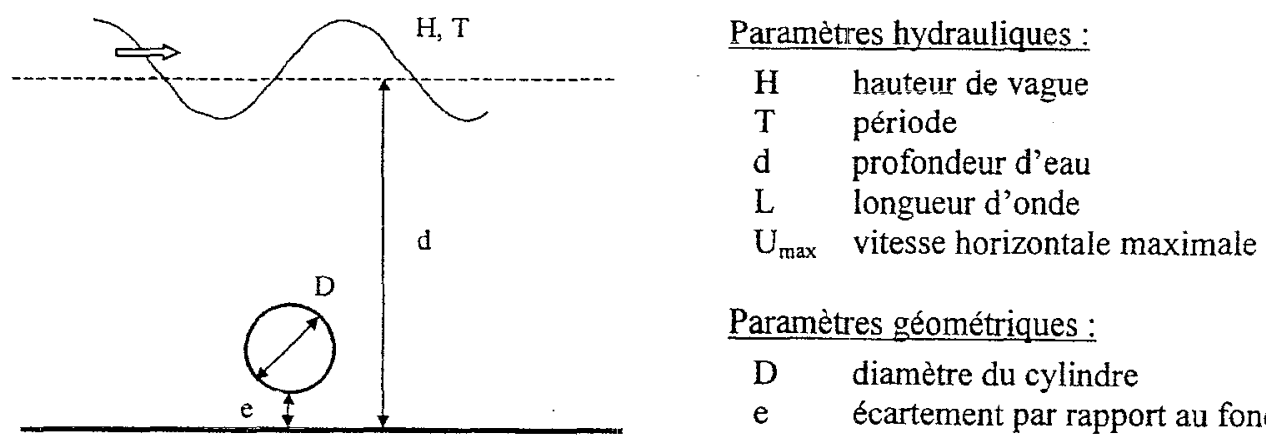

Paramètres géométriques:
D $\quad$ diamètre du cylindre
e

Figure 1 : Notations

Notations

Les efforts induits par la houle sur un cylindre horizontal près du fond vont être influencés par différents paramètres suivants (voir Figure 1 pour les principales 
notations) :

- Nombre de Keulegan et Carpenter $\mathrm{KC}=\mathrm{U}_{\max } \mathrm{T} / \mathrm{D}$

- Nombre de Reynolds $\mathrm{Re}=\mathrm{U}_{\max } \mathrm{D} / \mathrm{v}$

- Ecartement relatif par rapport au fond e/D

- Profondeur relative $\mathrm{d} / \mathrm{L}$

Parmi la multitude de publications sur le sujet, l'effort horizontal ou en ligne par unité de longueur $\mathrm{F}_{\mathrm{H}}$ est couramment modélisé en utilisant la formule de Morison (1950):

$\mathrm{F}_{\mathrm{H}}(\mathrm{t})=\frac{1}{2} \rho D \mathrm{C}_{\mathrm{D}}|\mathrm{U}(\mathrm{t})| \mathrm{U}(\mathrm{t})+\rho \frac{\pi \mathrm{D}^{2}}{4} \mathrm{C}_{\mathrm{M}} \frac{\partial \mathrm{U}(\mathrm{t})}{\partial \mathrm{t}}$

alors que les extremums de l'effort vertical ou de portance $F_{V}$ sont rapportés aux coefficients suivants :

$F_{\mathrm{V}, \max }=\frac{1}{2} \rho \mathrm{D} \mathrm{C}_{\mathrm{L}, \max } \mathrm{U}_{\max }^{2}$

$\mathrm{F}_{\mathrm{V}, \min }=\frac{1}{2} \rho \mathrm{DC}_{\mathrm{L}, \min } \mathrm{U}_{\max }^{2}$

$C_{D}, C_{M}$ et $C_{L, \text { max }}$ et $C_{L}$, min sont respectivement les coefficients de trainée, d'inertie et de portance maximum et minimum.

L'influence de la paroi est en particulier étudiée par Sarpkaya (1976) et Ali et Narayanan (1986). La gamme de nombre de Keulegan-Carpenter est intermédiaire (entre 5 et 25) dans les deux cas et correspond à celles de nos essais. Ces deux études montrent une croissance puis décroissance du coefficient de traînée $C_{D}$ lorsque le nombre de Keulegan-Carpenter $\mathrm{KC}$ augmente. Pour des valeurs croissantes de l'écartement relatif $e / D$, on note une diminution du coefficient d'inertie $C_{M}$. L'évolution du coefficient de traînée $C_{D}$ est mal définie pour les faibles écartements relatifs $e / D$. Le coefficient de portance maximum $C_{L}$, max diminue quand $\mathrm{KC}$ augmente et quand $\mathrm{e} / \mathrm{D}$ augmente.

L'influence de la profondeur sur les efforts exercés sur une conduite a fait l'objet de rares études. Selon Cheong et al (1987), les coefficients de traînée $C_{D}$ et d'inertie $\mathrm{C}_{\mathrm{M}}$ augmentent quand la profondeur $\mathrm{d}$ augmente à $\mathrm{KC}$ fixé. Il en va de même pour les coefficients de portance selon les mêmes auteurs.

Les années 90 voient l'apparition des premières études publiées traitant de l'influence du déferlement sur un cylindre horizontal. Endresen et Tørum (1992) mettent en évidence l'existence de forces élevées, de nature intermittente, induites par les déferlements plongeants. Ces efforts doivent être associés à la très forte intensité turbulente causée par le déferlement mais aucune mesure de celle-ci n'est présentée par les auteurs. Yüksel et Narayanan (1994a) ont également mené une étude en canal à houle sur les efforts exercés par la houle déferlante sur des cylindres horizontaux près d'un fond en pente. Ils montrent en particulier que les forces les plus importantes ont lieu lorsque le cylindre est positionné au point de plongée du déferlement, l'amplitude pouvant atteindre quatre fois celle mesurée au point de déferlement. Dans un second papier, Yüksel et Narayanan (1994b) calculent les efforts hydrodynamiques en utilisant la théorie cnoïdale pour estimer les vitesses dans la zone de déferlement et les coefficients hydrodynamiques 
obtenus par Ali et Narayanan (1986) en tunnel oscillant. Les pics d'efforts horizontaux et verticaux ainsi calculés sont en accord avec les mesures expérimentales présentées par ailleurs.

Dans le cadre d'un programme de recherche sur la stabilisation des conduites sous-marines en zone côtière (Chevalier, 2000), nous avons réalisé des mesures d'efforts sur un cylindre horizontal près du fond. La description de ces essais est donnée dans la partie suivante. Les efforts sont ensuite présentés et une modélisation de l'effort en ligne selon le modèle de Morison est enfin menée et discutée.

\section{Présentations des essais}

\subsection{Dispositif expérimental}

Cette étude expérimentale a été menée dans le canal à houle du laboratoire (longueur $22 \mathrm{~m}$, largeur $0,8 \mathrm{~m}$ ). Une plage de $10 \mathrm{~m}$ de longueur, de pente constante égale à $5 \%$, est installée à l'extrémité du canal afin de simuler la déformation de la houle lors de sa propagation vers la côte.

Un cylindre (diamètre $\mathrm{D}=40 \mathrm{~mm}$, longueur $\mathrm{l}=77 \mathrm{~cm}$ ) équipé de jauges internes d'extensométrie dans sa partie centrale a été mis en œuvre, les signaux provenant des modules étant enregistrés sur un ordinateur. Le cylindre est fixé aux parois latérales du canal et un étalonnage horizontal et vertical est effectué à l'aide de masses marquées avant chaque série de mesures.

Dans le même temps, plusieurs sondes de surface libre nous renseignent sur l'évolution de la surface libre. Trois sondes S1, S2 et S3 placées à l'amont permettent de déterminer les paramètres de la houle incidente. Les sondes S4 à S7 sont réparties le long de la plage de déferlement et permettent de quantifier l'évolution de la surface libre (voir Figure 2). La sonde S6, située à l'aplomb du cylindre, donne l'évolution du profil temporel de surface libre $\eta(\mathrm{t})$.

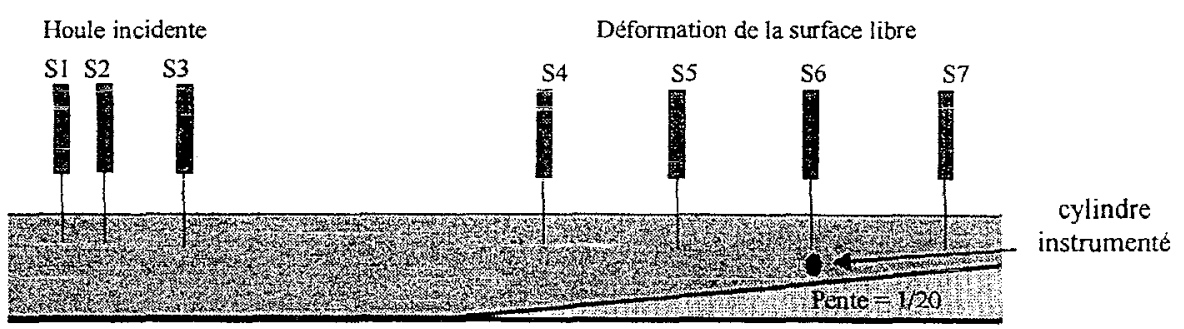

Figure 2 : Dispositif expérimental Experimental set-up

\subsection{Conditions expérimentales}

Les essais ont été réalisés en houle régulière. La période pour tous ces essais a été de $1,8 \mathrm{~s}$. Nous avons fait varier la hauteur de houle incidente $H_{i}$ depuis les faibles houles jusqu'à la limite possible du batteur $\left(\mathrm{H}_{\mathrm{i}}=2\right.$ à $\left.14 \mathrm{~cm}\right)$.

Deux profondeurs d'eau ont été testées, respectivement $42 \mathrm{~cm}$ et $37 \mathrm{~cm}$ au large (soit d $=25 \mathrm{~cm}$ et $20 \mathrm{~cm}$ au droit du cylindre). Dans ces deux configurations le déferlement a toujours eu lieu à l'aval du cylindre. Nous nous trouverons ainsi, dans tous les essais, en présence de houle pré-déferlante et non en présence de houle déferlante. 
Différentes configurations géométriques ont été testées au cours de cette étude correspondant à une gamme d'écartements relatifs e/D compris entre 0 et 0,5 .

\subsection{Estimation de la composante horizontale de la vitesse orbitale}

Que ce soit pour définir le régime d'écoulement $(\mathrm{KC}, \mathrm{Re})$ ou pour déterminer les coefficients hydrodynamiques d'efforts, une connaissance préalable de l'écoulement est nécessaire. En l'absence de mesures expérimentales des vitesses lors des essais avec le cylindre instrumenté, nous avons employé un modèle empirique développé par Koyama et Iwata (1986) nommé MTFM par la suite (pour Modified Transfer Function Method).

Le calcul de la vitesse est réalisé à partir de la dénivelée $\eta(t)$ et d'une fonction de transfert par la relation suivante :

$$
\mathrm{U}(\mathrm{t})=\mathrm{H}_{\mathrm{MTFM}}(\mathrm{d}, \mathrm{T}, \mathrm{s}) \eta(\mathrm{t})
$$

La fonction de transfert $H_{\text {MTFM }}(d, T, s)$ est définie par :

$$
\left\{\begin{array}{l}
\eta(t)>0 \Rightarrow H_{M T F M}(d, T, s)=\frac{2 \pi}{T} \frac{\cosh (k s)}{\sinh \left(d+\eta(t)\left(1-\frac{s}{d+\eta(t)}\right)\right)} \\
\eta(t) \leq 0 \Rightarrow H_{M T F M}(d, T, s)=\frac{2 \pi}{T} \frac{\cosh (k s)}{\sinh (k d)}
\end{array}\right.
$$

où s représente l'élévation par rapport au fond et $\mathrm{k}$ le nombre d'onde calculé par la théorie linéaire.

Outre les résultats présentés par Koyama et Iwata, cette méthode a été validée de façon satisfaisante près du fond sur notre dispositif expérimental pour une très large gamme de houles de peu déformées à limite déferlantes (Chevalier, 2000). Nous utiliserons donc cette technique d'estimation de vitesse pour l'analyse de nos mesures d'efforts. Appliquée à nos conditions expérimentales, l'estimation de la vitesse maximale par la méthode MTFM conduit à une variation du nombre de Keulegan et Carpenter comprise entre 4 et $19\left(3.10^{4}<\operatorname{Re}<1,8.10^{5}\right)$.

\section{Résultats des mesures d'efforts}

\subsection{Efforts extrêmes}

Les figures $3 \mathrm{a}$ à $3 \mathrm{e}$ ci-après présentent les efforts horizontaux et verticaux maximaux et minimaux en fonction de la hauteur de houle pour différents écartements relatifs dans le cas $d=20 \mathrm{~cm}$. On remarque logiquement une croissance régulière des différents efforts extrêmes en fonction de la hauteur de houle. Par ailleurs, on note une forte dissymétrie des pics positifs $F_{\mathrm{H}}$, max et négatifs $\mathrm{F}_{\mathrm{H} \text {, min }}$ de l'effort en ligne pour les houles les plus déformées.

Une forte décroissance de l'effort vertical positif $\mathrm{F}_{\mathrm{V} \text {, max }}$ (opposé à la paroi) en fonction de l'écartement relatif est aussi à relever. Elle est nettement visible de $\mathrm{e} / \mathrm{D}=0$ à $\mathrm{e} / \mathrm{D}=0,25$. Pour les valeurs supérieures, il existe une stabilisation $\mathrm{du}$ phénomène. Les efforts de portance minima $F_{V}$, min sont très faibles voire nuls pour les trois premiers écartements. En augmentant e/D, ces efforts minimums deviennent franchement négatifs (orientés vers la paroi). Les efforts horizontaux montrent eux une faible sensibilité vis-à-vis de l'écartement cylindre - paroi. 
a) $\mathrm{e} / \mathrm{D}=0$
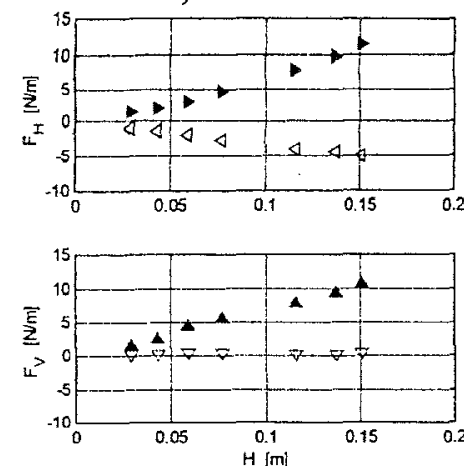

d) $\mathrm{e} / \mathrm{D}=0,375$
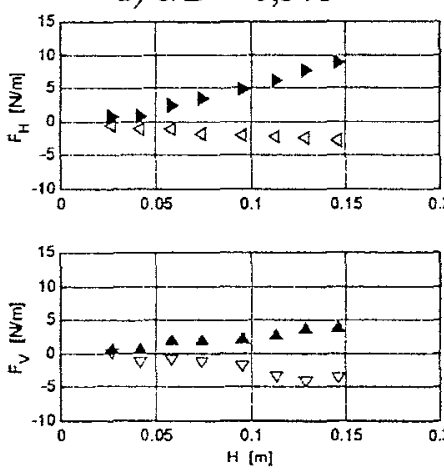

b) $\mathrm{e} / \mathrm{D}=0,125$
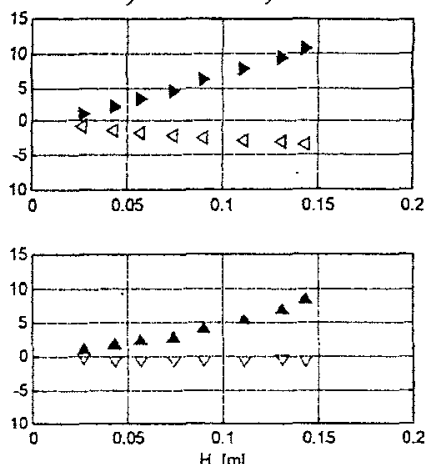

e) $\mathrm{e} / \mathrm{D}=0,5$
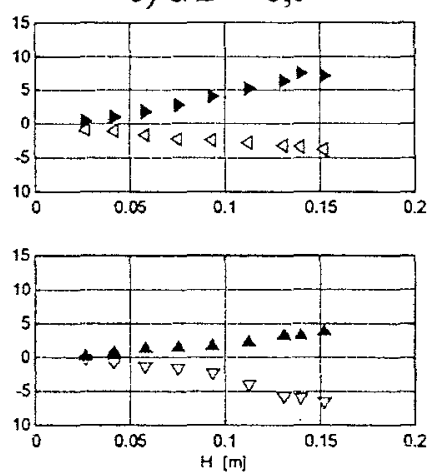

c) $\mathrm{e} / \mathrm{D}=0,25$
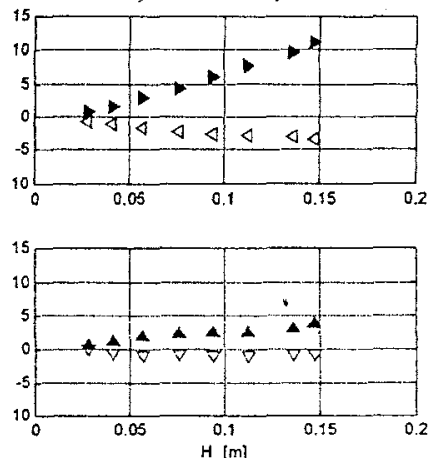

Définition des symboles

$-\mathrm{F}_{\mathrm{H}, \max } \triangleleft \mathrm{F}_{\mathrm{H}, \min }$

$\Delta \mathrm{F}_{\mathrm{V}, \max } \quad \nabla \mathrm{F}_{\mathrm{V}, \min }$

cas $\mathrm{d}=20 \mathrm{~cm}$

Figure 3 : Efforts extrêmes en fonction de la hauteur de houle Extreme forces versus wave height

\subsection{Evolution temporelle des efforts}

Les graphiques de la figure 4 présentent l'évolution temporelle des efforts mesurés pour quelques-unes des configurations testées à la profondeur $\mathrm{d}=20 \mathrm{~cm}$. Ces efforts ont été normalisés de la façon suivante, $\mathrm{U}_{\max }$ étant calculé par la méthode MTFM :

$$
\begin{aligned}
& C_{\mathrm{H}}(\mathrm{t})=\frac{F_{\mathrm{H}}(\mathrm{t})}{\frac{1}{2} \rho D U_{\max }^{2}} \\
& \mathrm{C}_{\mathrm{V}}(\mathrm{t})=\frac{\mathrm{F}_{\mathrm{V}}(\mathrm{t})}{\frac{1}{2} \rho D U_{\max }^{2}}
\end{aligned}
$$

Aux faibles KC, l'effort de portance présente une fréquence double de celle de la houle, pour tous les écartements testés. En augmentant le nombre de Keulegan et Carpenter, de nouveaux maxima apparaissent, plus ou moins marqués selon l'éloignement du cylindre à la paroi.

L'effort de portance est très sensible à l'écartement. Pour de faibles e/D, l'effort est majoritairement positif $\left(\mathrm{F}_{\mathrm{V}}>0\right.$ à tout instant pour $\left.\mathrm{e} / \mathrm{D}=0\right)$. Le blocage du fluide en partie basse induit des pressions sous le cylindre supérieures à celles générées dans sa partie haute. La différence de pression est d'autant plus importante que la vitesse au-dessus du cylindre est importante. Le passage de la crête sera donc associé à un pic de portance positif et de faible durée.

En écartant le cylindre $(\mathrm{e} / \mathrm{D}=0,25)$, l'effort vertical se recentre alors sur la valeur 
nulle. Le passage du fluide sous le cylindre est possible mais perturbé. Les vitesses sous le cylindre sont alors inférieures à celles au-dessus. Cette dissymétrie induit le même type d'effort que précédemment. Les valeurs des pics de portance sont cependant diminuées. En effet, la différence de vitesse entre les parties supérieures et inférieures du cylindre s'amenuise. Les effets de portance associés à cette différence sont alors de plus en plus réduits.

Pour $\mathrm{e} / \mathrm{D}=0,5$ on note au contraire un phénomène d'accélération du fluide sous le cylindre au passage de la crête induisant un pic de portance orienté vers la paroi.

a) Profils de surface libre - Cas $d=20 \mathrm{~cm}$
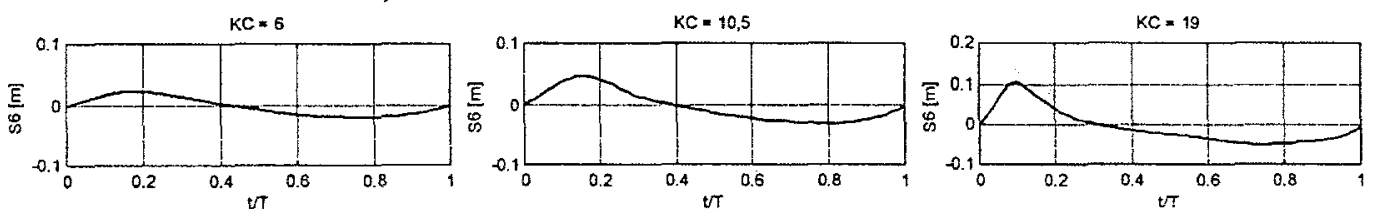

b) Efforts pour e $/ D=0$
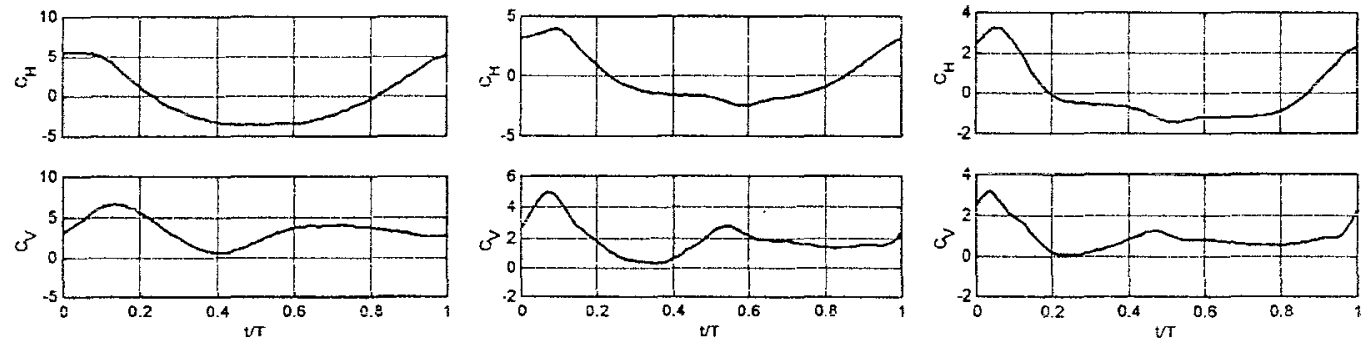

c) Efforts pour e $/ D=0,25$
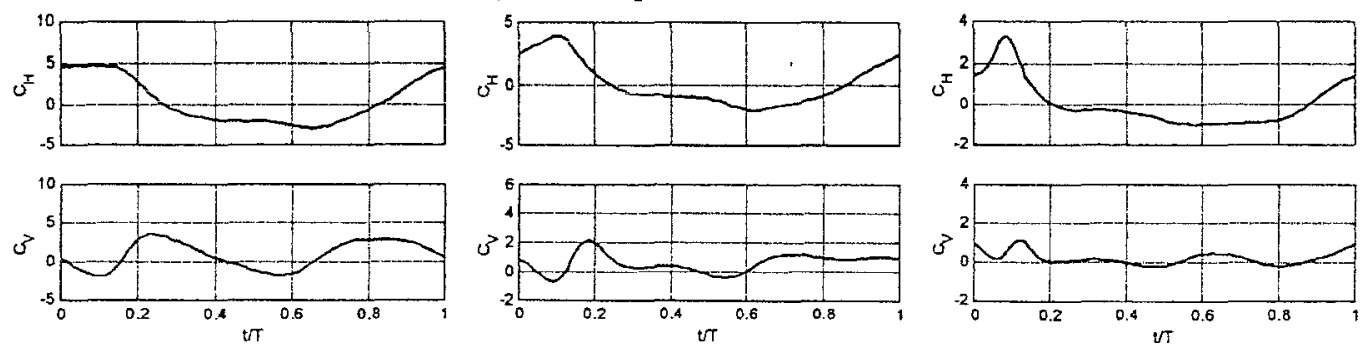

d) Efforts pour e $/ \mathrm{D}=0,5$
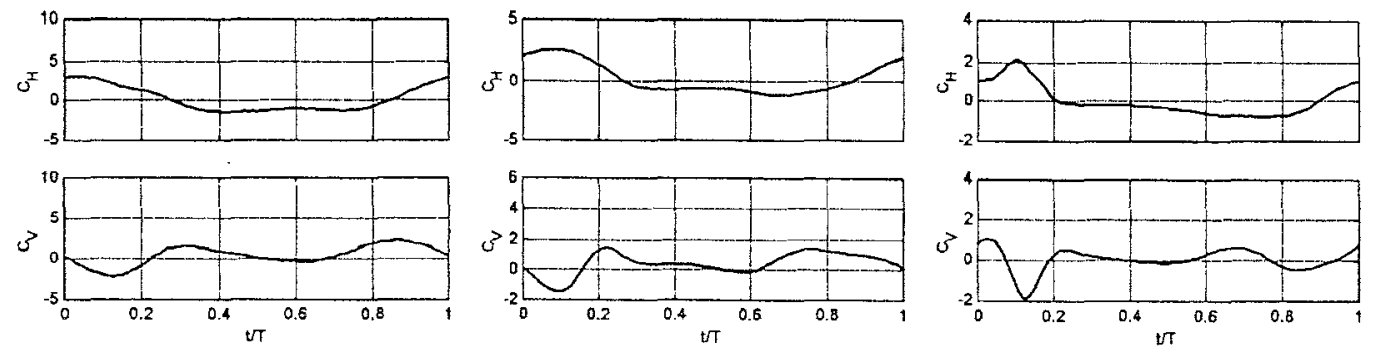

Figure 4 : Evolution temporelle du profil de surface libre et des efforts normalisés

Temporal evolution of wave profile and normalised forces

En ce qui concerne l'effort en ligne, on trouve classiquement un effort dont l'évolution est similaire à celle de la surface libre, bien que légèrement déphasé. Dans le cas d'un cylindre posé sur le fond, le pic d'effort horizontal lié au passage de la crête est simultané à un pic de portance positif ce qui peut conduire à une déstabilisation de la conduite. Pour les autres écartements, l'effort en ligne maximum correspond à un effort de portance minimum. 


\section{Analyse de l'effort en ligne}

Nous présentons dans cette partie les coefficients hydrodynamiques en ligne au sens de la formulation de Morison calculés à partir de nos mesures expérimentales d'efforts. La validité de cette formulation est également présentée et discutée pour deux modèles de calcul de la vitesse horizontale.

\subsection{Détermination des coefficients hydrodynamiques en ligne}

Le calcul des coefficients de traînée et d'inertie de la formule de Morison a été fait en utilisant la méthode des moindres carrés. La vitesse horizontale a été estimée à l'aide de la méthode MTFM. La validité de $C_{M}$ et $C_{D}$ peut être évaluée en recomposant l'effort à partir de la formule de Morison, et en le comparant à l'effort mesuré. On calcule ainsi le paramètre d'erreur quadratique relative $\delta$ :

$\delta=\frac{\int_{0}^{T}\left(\mathrm{~F}_{\mathrm{H}, \text { mesure }}-\mathrm{F}_{\mathrm{H} \text {, calcule }}\right)^{2} \mathrm{dt}}{\int_{\mathrm{H}, \text { mesuré }}^{\mathrm{T}} \mathrm{dt}}$

Une première analyse de nos efforts a montré une composante moyenne non nulle de l'effort en ligne pour les houles les plus fortes. Une décomposition selon la formulation classique de Morison prend mal en compte ce phénomène et conduit alors à des erreurs relatives $\delta$ importantes. Pour minimiser ce phénomène, nous avons introduit un paramètre $\mathrm{C}_{0}$, fonction du régime d'écoulement $\mathrm{KC}$, dans la formule de Morison. Celle-ci s'écrit alors sous la forme modifiée suivante :

$$
\mathrm{F}_{\mathrm{H}}(\mathrm{t})=\mathrm{C}_{0}+\frac{1}{2} \rho D \mathrm{C}_{\mathrm{D}}|\mathrm{U}(\mathrm{t})| \mathrm{U}(\mathrm{t})+\rho \frac{\pi \mathrm{D}^{2}}{4} \mathrm{C}_{\mathrm{M}} \frac{\partial \mathrm{U}(\mathrm{t})}{\partial \mathrm{t}}
$$

Une étude de l'influence de la prise en compte de $C_{0}$ sur les coefficients $C_{D}$ et $C_{M}$ a montré que ces deux derniers paramètres ne variaient pratiquement pas suivant la formulation de Morison employé (Eq. 3 ou Eq. 11).

\subsubsection{Coefficient de traînée}

La figure 5 présente l'évolution du coefficient de traînée en fonction du nombre de Keulegan et Carpenter, pour les deux profondeurs testées. L'allure globale de ces courbes semble conforme aux résultats de la littérature (Sarpkaya, 1976, Ali et Narayanan, 1986) : croissance de $\mathrm{C}_{\mathrm{D}}$ pour $\mathrm{KC}<7$, un maximum en 7 , puis décroissance.

A régime élevé $(\mathrm{KC}>10)$, l'écartement relatif e/D semble être de peu d'influence sur le coefficient de traînée, et ce pour les deux profondeurs testées. En revanche, l'effet de la profondeur est beaucoup plus net : une augmentation de la profondeur $\mathrm{d}$ conduit à des valeurs plus élevées de $\mathrm{C}_{\mathrm{D}}$. Ceci est en accord avec les résultats de Cheong et al. (1987).

\subsubsection{Coefficient d'inertie}

La figure 6 présente le coefficient d'inertie en fonction de la profondeur et du nombre de Keulegan et Carpenter. On peut tout d'abord noter que ce coefficient d'inertie augmente avec la profondeur d, résultat là encore conforme à ceux obtenus par Cheong et al. (1987).

L'influence de l'écartement relatif est ici beaucoup plus nette que pour le 
coefficient de traînée. Le coefficient d'inertie décroît lorsque l'écartement relatif augmente, phénomène déjà mis en évidence par Ali et Narayanan (1986). Cette décroissance semble se faire par paliers comme le prouve le regroupement des courbes e/D $=0,125$ et 0,25 d'une part, e/D=0,375 et 0,5 d'autre part. On peut rapprocher ces trois tendances des trois types de comportements mis en évidence lors des visualisations menées par ailleurs (Chevalier, 2000).

a) $\mathrm{d}=25 \mathrm{~cm}$

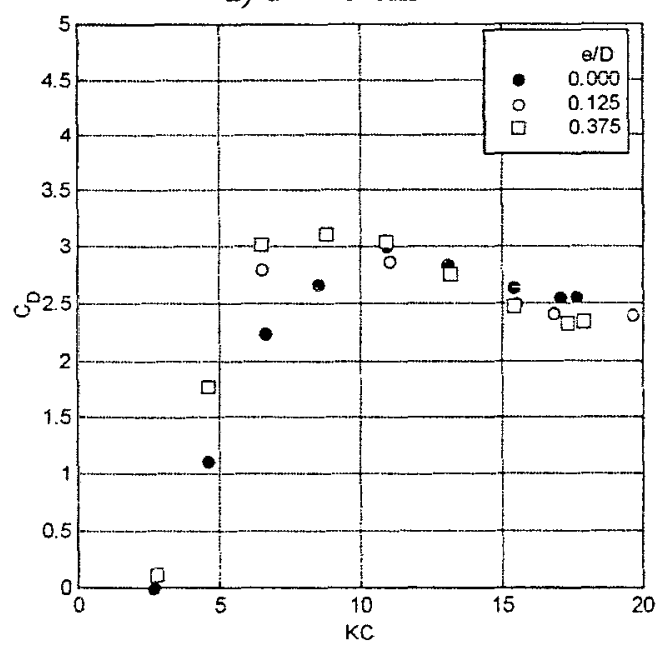

b) $\mathrm{d}=20 \mathrm{~cm}$

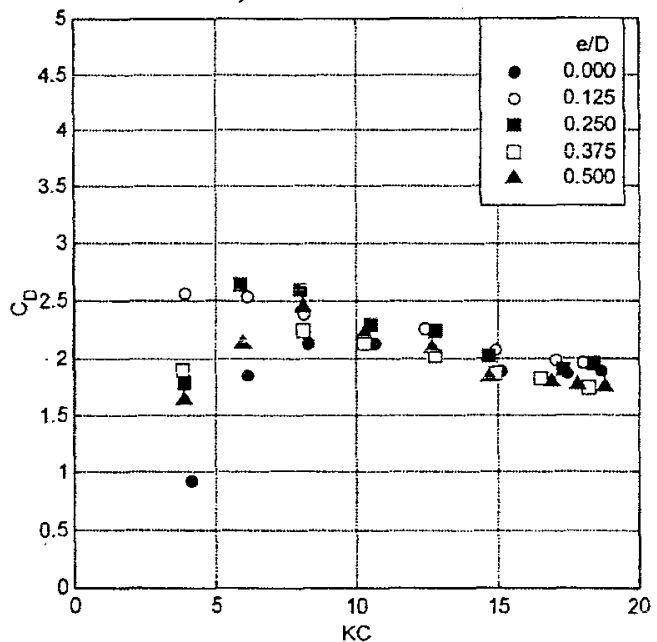

Figure 5 : Coefficient de traînée $C_{D}$ en fonction du nombre de Keulegan-Carpenter $\mathrm{KC}$ Drag coefficient $C_{D}$ versus Keulegan-Carpenter number $K C$

a) $\mathrm{d}=25 \mathrm{~cm}$

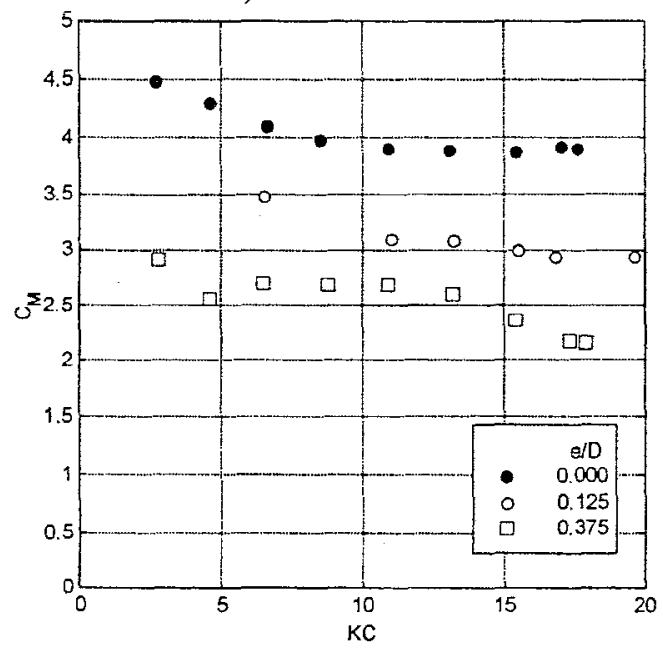

b) $\mathrm{d}=20 \mathrm{~cm}$

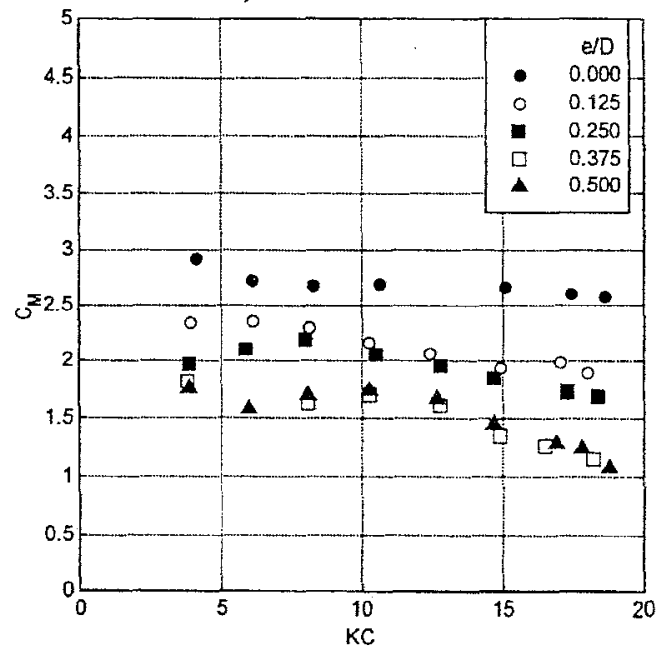

Figure 6 : Coefficient d'inertie $C_{M}$ en fonction du nombre de Keulegan-Carpenter KC Inertia coefficient $C_{M}$ versus Keulegan-Carpenter number $K C$

\subsubsection{Coefficient Co}

Le calcul de $C_{M}$ et $C_{D}$ a été fait en considérant une formulation de Morison quelque peu modifiée (Eq. 11), dans laquelle une composante moyenne $\mathrm{C}_{0}$ est introduite. L'évolution de ce paramètre avec $\mathrm{KC}$ est présentée ci-après (Figure 7). Dans les deux cas de profondeurs, on note une forte croissance du coefficient $\mathrm{C}_{0}$ avec le nombre de Keulegan et Carpenter. Ce résultat semble logique, dans la 
mesure où la déformation (dissymétrie) de la vague augmente avec $\mathrm{KC}$.

a) $\mathrm{d}=25 \mathrm{~cm}$

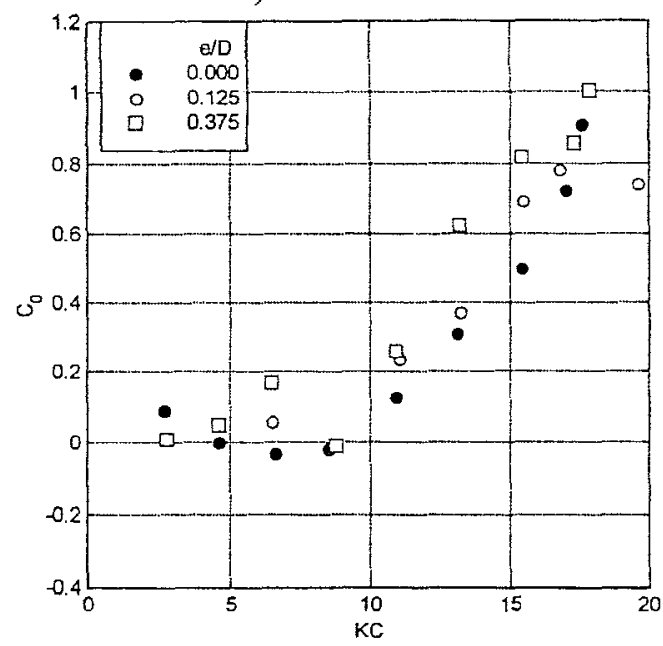

b) $\mathrm{d}=20 \mathrm{~cm}$

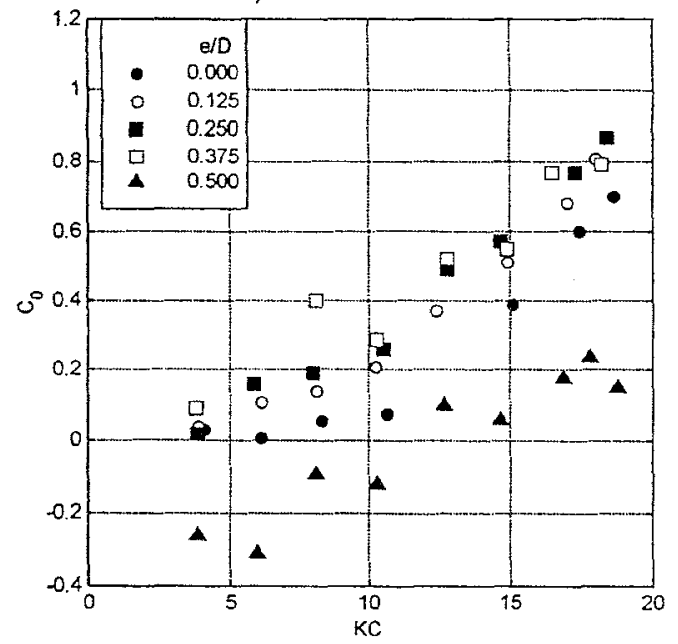

Figure 7 : Coefficient $\mathrm{C}_{0}$ en fonction du nombre de Keulegan-Carpenter $\mathrm{KC}$ Coefficient $C_{0}$ versus Keulegan-Carpenter number $K C$

\subsection{Recomposition de l'effort en ligne}

Divers moyens ont été utilisés pour tester la validité de nos coefficients. Le principe est de recomposer l'effort en ligne, à partir de nos valeurs de $C_{M}, C_{D}$ et $\mathrm{C}_{0}$. On peut alors :

- calculer le paramètre d'erreur $\delta$ sur une période (Eq. 10);

- comparer les évolutions temporelles de l'effort calculé et de l'effort mesuré ;

- comparer les valeurs extrêmes des efforts mesurés et prédits.

L'objet de cette partie est de présenter ces tests de validation. Une comparaison a été également réalisée avec des essais de calcul complets (détermination des coefficients hydrodynamiques et recomposition des efforts) utilisant la théorie linéaire d'Airy pour estimer la vitesse au lieu de la méthode MTFM.

\subsubsection{Estimation de l'erreur globale}

D'une façon générale, l'erreur $\delta$ augmente avec le nombre de Keulegan et Carpenter pour les deux profondeurs testées. La figure 8 présente les résultats obtenus en utilisant la théorie linéaire et la méthode MTFM pour le cas $d=20 \mathrm{~cm}$. $\mathrm{La}$ variation de l'erreur avec $\mathrm{KC}$ est globalement linéaire. Il est cependant évident que la méthode d'estimation par la théorie linéaire induit des erreurs plus fortes que celles obtenues avec la MTFM. Pour tous les écartements relatifs, l'estimation des vitesses par la Méthode de la Fonction de Transfert Modifiée aboutit à des valeurs de $\delta$ beaucoup plus faibles. De plus cette méthode ne semble pas surestimer la valeur du nombre de Keulegan et Carpenter, c'est à dire la vitesse maximale de l'écoulement (Chevalier, 2000).

Pour les calculs menés en estimant la vitesse horizontale par l'estimation MTFM, l'erreur relative est supérieure à $10 \%$ pour des nombres de Keulegan-Carpenter supérieurs à environ 12. Cette gamme de $\mathrm{KC}$ correspond à celle où la formulation de Morison est peu représentative de l'effort réel (Sarpkaya et Isaacson, 1981). 
Nous ne disposons pas de mesures pour les $\mathrm{KC}>20-30$ pour conclure sur la validité du modèle et de notre démarche dans le domaine de traînée.

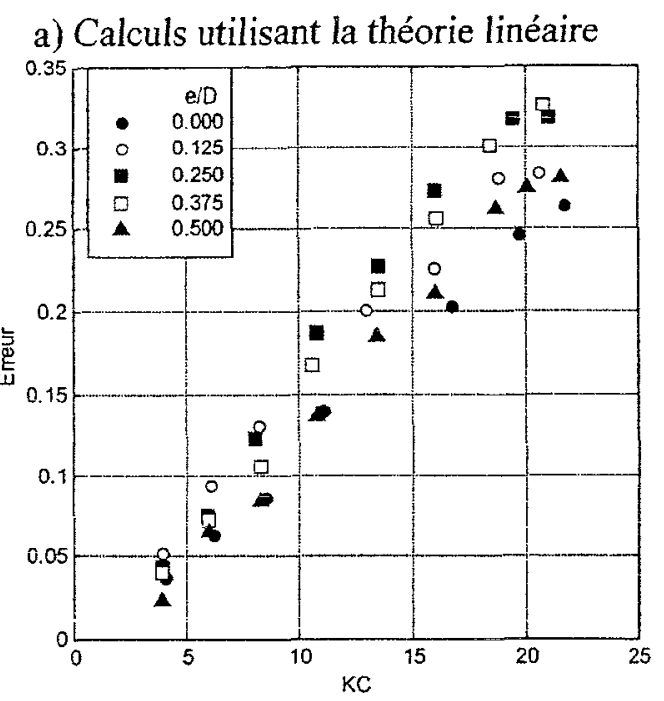

b) Calculs utilisant l'estimation MTFM

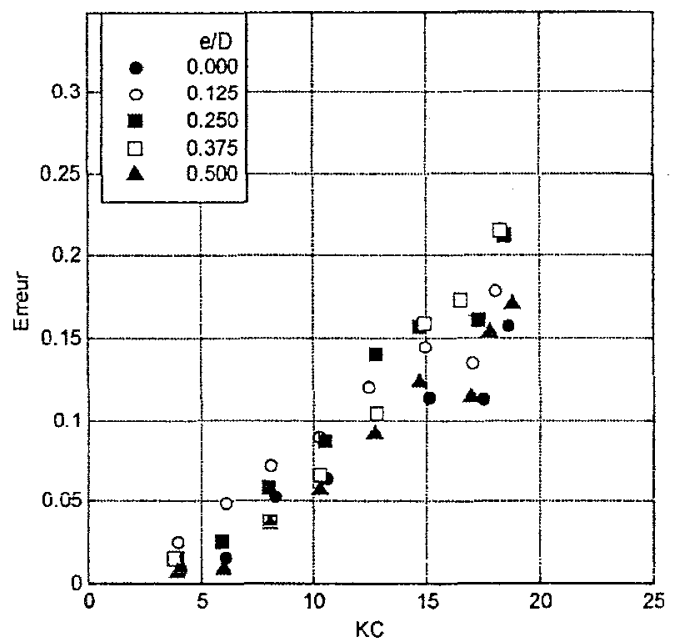

Figure 8: Erreur relative $\delta$ fonction du nombre de Keulegan-Carpenter $\mathrm{KC}(\mathrm{d}=20 \mathrm{~cm})$ Relative error $\delta$ versus Keulegan-Carpenter number $K C(d=20 \mathrm{~cm})$

\subsubsection{Allure sur une période}

La figure 9 présente une comparaison temporelle des efforts mesurés et des efforts calculés pour différents nombres de Keulegan-Carpenter, en utilisant pour les vitesses soit le modèle d'Airy, soit la méthode MTFM. Le calcul utilisant la méthode MTFM est le plus proche de la courbe des efforts mesurés. Dans les deux premiers cas, on note même une assez bonne concordance entre l'effort recomposé et l'effort mesuré contrairement au modèle d'Airy.

a) $\mathrm{KC}=6$

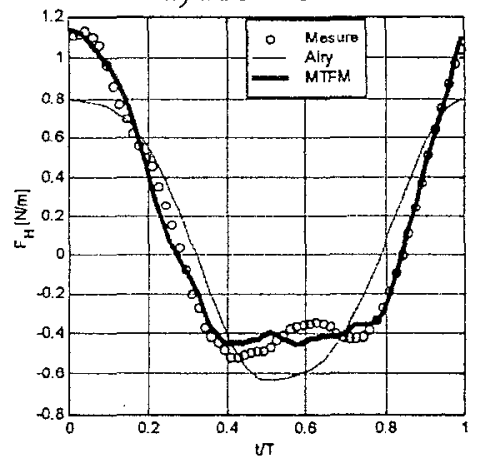

b) $\mathrm{KC}=10,5$

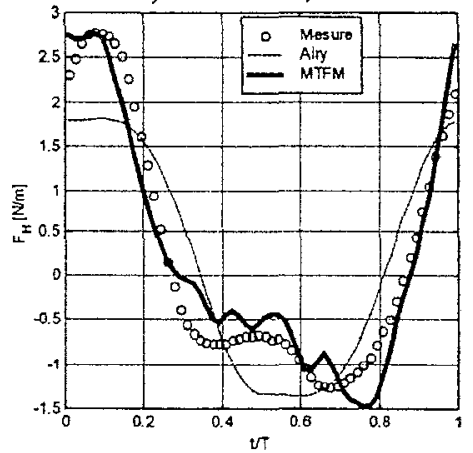

c) $\mathrm{KC}=19$

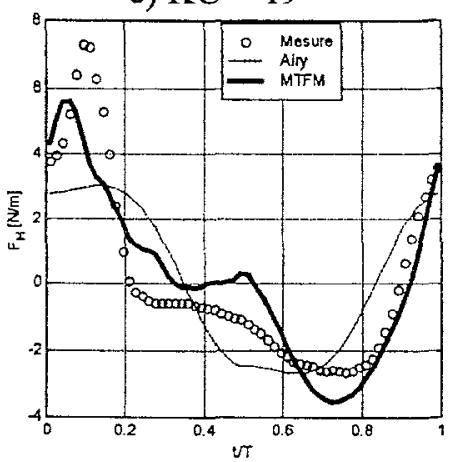

Figure 9 : Exemples de modélisation de l'effort en ligne $(\mathrm{e} / \mathrm{D}=0,5$ et $\mathrm{d}=20 \mathrm{~cm})$ Examples of modelling of in-line force $(e / D=0,5$ and $d=20 \mathrm{~cm})$

L'exemple de la figure précédente montre clairement que la théorie linéaire est défaillante. Le pic d'effort maximal est très largement sous-estimé par cette théorie. Au contraire, la méthode MTFM montre ici son intérêt pour les nombres de Keulegan-Carpenter intermédiaires. Pour le plus grand KC (Figure 9-c), les deux théories sont incapables de représenter l'effort en ligne mais on touche là aux limites de la formulation de Morison (Sarpkaya et Isaacson, 1981). 


\subsubsection{Reproduction des pics d'efforts}

Pour un bon dimensionnement, l'ingénieur concepteur doit être à même de déterminer l'effort maximal auquel la structure sera soumise. Nous avons donc comparé les pics d'efforts mesurés à ceux des efforts calculés pour les deux méthodes de calculs considérées et la profondeur $\mathrm{d}=20 \mathrm{~cm}$ (Figure 10).

Les valeurs des efforts extrêmes prédits par la théorie linéaire sont d'une façon générale sous-estimées. En effet, les résultats de cette méthode ne coïncident avec les mesures que dans une plage réduite de valeurs (entre environ -4 et $+2,5 \mathrm{~N} / \mathrm{m}$ ). Les efforts négatifs de l'ordre de $-5 \mathrm{~N} / \mathrm{m}$ sont sous évalués. Les pics d'efforts positifs sont eux très largement sous-évalués par la théorie linéaire. En effet pour les valeurs les plus fortes, la mesure représente jusqu'au double de la valeur prédite. L'écart entre mesure et calcul est croissant et semble d'ailleurs linéaire, au moins dans notre gamme d'essais. Il apparaît donc que la prévision des pics d'efforts par une théorie linéaire conduit à nettement les sous-estimer. Cette constatation nous conduit à assurer qu'il serait dangereux de pré-dimensionner une structure en ne se basant que sur cette approche.

a) Théorie linéaire

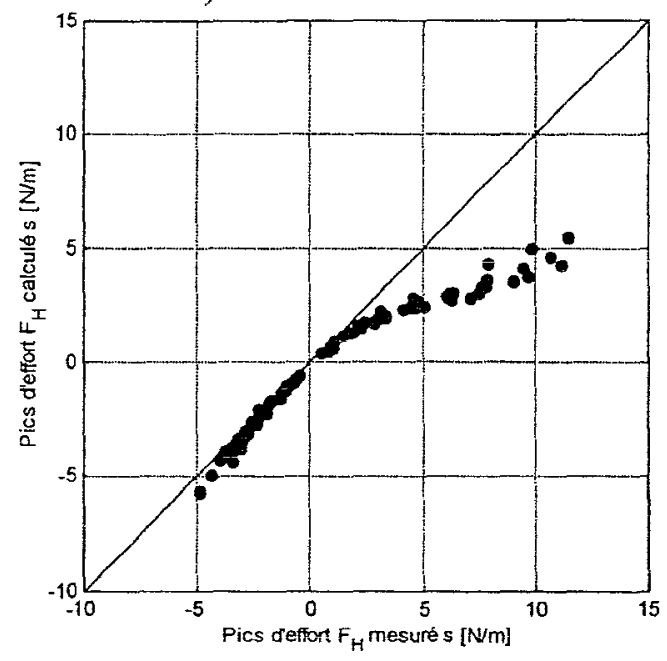

b) Estimation MTFM

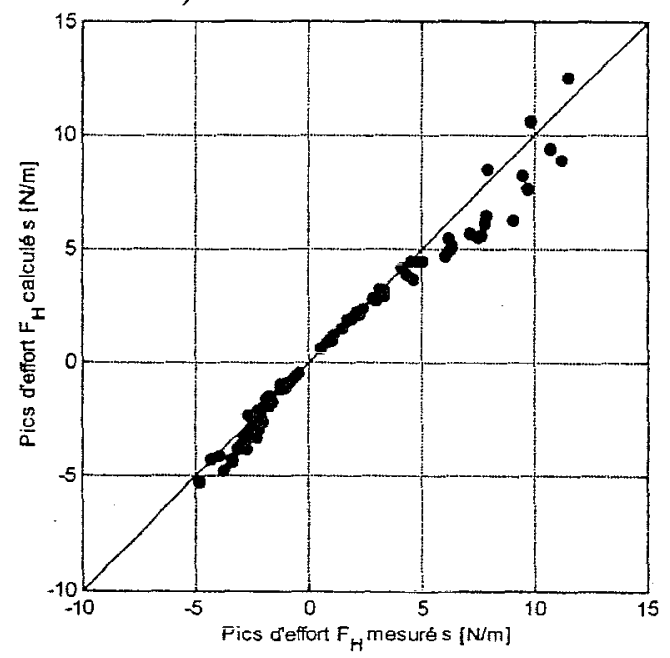

Figure 10 : Comparaison entre pics d'effort horizontal mesurés et calculés $(\mathrm{d}=20 \mathrm{~cm})$ Comparison between measured and calculated maximum of horizontal forces $(d=20 \mathrm{~cm})$

La comparaison des efforts extrêmes prédits en utilisant l'estimation MTFM et ceux mesurés expérimentalement est présentée sur la figure 10-b). Globalement les résultats concordent mieux qu'avec la précédente méthode de calcul. L'analyse de l'effort en ligne à l'aide de l'estimation MTFM conduit à des résultats plus réalistes quant à la détermination des efforts maximaux. Les valeurs des pics négatifs sont très légèrement sous-estimées, moins toutefois que par la théorie linéaire. Le bénéfice est surtout marqué pour les pics d'efforts positifs, les écarts moyens sont de l'ordre de $10 \%$, conduisant parfois à légèrement sousestimer ou au contraire légèrement surévaluer l'effort maximum résultant. L'écart reste donc raisonnable et démontre la bonne adéquation entre l'approche théorique et la réalité de l'écoulement engendré par la plage si on prend bien soin d'estimer correctement la vitesse horizontale. 


\section{Conclusions}

Nous avons mené une étude expérimentale des efforts induits par la houle sur un cylindre près du fond en zone côtière. Nous avons ainsi notamment mis en évidence l'influence de la dissymétrie de l'écoulement de houle sur les efforts et l'importance de sa prise en compte pour l'analyse de l'effort en ligne au sens de Morison. Ceci se fait notamment par l'utilisation d'un modèle de vitesse simplifié qui permet de valider la formulation de Morison aux cas des houles prédeferlantes pour des nombres de Keulegan- Carpenter intermédiaires (KC compris entre 4 et environ 12). Le calcul des pics de l'effort horizontal par cette méthode conduit à une assez bonne concordance avec les pics mesurés.

Les essais réalisés se sont limités à quelques conditions expérimentales et ne prétendent pas couvrir un large éventail de situations réelles. En cela ce travail se veứ une étude originale des efforts engendrés par une houle pré-déferlante et non une tentative de mise en œuvre d'une méthode de dimensionnement d'un pipeline. En effet, la modélisation effectuée reste perfectible aussi bien par l'extension des capacités du dispositif expérimental employé (autres profondeurs, plus grands $\mathrm{KC}$, prise en compte du déferlement) que par les problèmes potentiels d'effet d'échelle.

\section{Références}

- Ali N., Narayanan R. (1986) "Forces on cylinders oscillating near a plane boundary", Proceedings of the $5^{\text {th }}$ International Conference on Offshore Mechanics and Artic Engineering, Tokyo, Vol. III, pp 613-619.

- Cheong H.F., Jothi Shankar N., Subbiah K. (1987) "Wave forces on submarine pipelines near a plane boundary", Ocean Engineering, Vol. 14, No. 3 , pp 181-200.

- Chevalier C. (2000) "Action de la houle sur un cylindre immergé dans la zone côtière", Thèse de Doctorat, Université de Caen, 184 p.

- Endresen H.K., Tørum A. (1992) "Wave forces on a pipeline through the surf zone", Coastal Engineering, Vol. 18, pp 267-281.

- Koyama H., Iwata K. (1986) "Estimation of water particle velocities of shallow water waves by a modified transfer function method", Proceedings of the International Conference on Coastal Engineering, pp 425-436.

- Morison J.R., O'Brien M.P., Johnson J.W., Schaaf S.A. (1950) "The force exerted by surface waves on piles", Petroleum Transactions, AIME, Vol. 189, pp 149-154.

- Sarpkaya T. (1976) "Forces on cylinders near a plane boundary in a sinusoidally oscillating fluid", Journal of Fluids Engineering, September 76, pp 499-505.

- Sarpkaya T., Isaacson M. (1981), "Mechanics of wave forces on offshore structures", Van Nostrand Reinhold Company, 651 p.

- Yüksel Y., Narayanan R. (1994a) "Breaking wave forces on horizontal cylinder close to the seabed", Coastal Engineering, Vol. 23, pp 115-133.

- Yüksel Y., Narayanan R. (1994b) "Wave forces on horizontal cylinder resting on sloping bottom", Proceedings of the 4th International Offshore and Polar Engineering Conference, Osaka, Japon, Vol. II, pp 85-92. 